\title{
Sagnac interference in carbon nanotubes
}

\author{
Waheb Bishara, Gil Refael, and Marc Bockrath \\ Department of Physics, California Institute of Technology, Pasadena, California 91125, USA
}

(Received 20 June 2008; revised manuscript received 17 September 2008; published 6 October 2008)

\begin{abstract}
The Sagnac interference mode arises when two interfering counterpropogating beams traverse a loop, but with their velocities detuned by a small amount $2 u$, with $v_{R / L}=v_{F} \pm u$. In this paper we perform a perturbative nonequilibrium calculation of Sagnac interference in single-channel wires as well as armchair nanotube loops. We study the dependence of the Sagnac conductance oscillations on temperature and interactions. We find that the Sagnac interference is not destroyed by strong interactions, but becomes weakly dependent on the velocity detuning $u$. In armchairs nanotubes with typical interaction strength, $0.25 \leq g \leq 0.5$, we find that the necessary temperature for observing the interference effect, $T_{\mathrm{SAG}}$ is also only weakly dependent on the interaction, and is enhanced by a factor of 8 relative to the temperature necessary for observing Fabry-Pérot interference in the same system, $T_{\mathrm{FP}}$.
\end{abstract}

DOI: 10.1103/PhysRevB.78.165405

PACS number(s): 73.63.Fg

\section{INTRODUCTION}

One of the most tantalizing effects predicted by quantum mechanics is the appearance of interference fringes when two matter beams come together. These fringes provided the ultimate testimony to the pertinence of quantum mechanics and the Schrödinger equation. Interferometry of light is employed in many precision measurement devices. The MachZehnder interferometer produces interference between two beams traversing two distinct paths, one of which passes through a test chamber containing, for instance, a dilute gas (see Fig. 1); this setup was originally used to measure the refraction index of the gas in the chamber. Fabry-Pérot interferometer recombines a series of beams, where the $n$th beam traverses the optical path between two mirrors or through a loop $n$ times. The narrowness of the resulting interference peaks allows a precise measurement of a light beam's wavelength, and is commonly used to measure the Zeeman splitting of an atom in a magnetic field. The most sensitive of all interference constructs, however, is the Sagnac interference. ${ }^{1}$ In this setup, a light (or matter ${ }^{2}$ ) beam is split into two beams, which traverse the interferometer's loop both clockwise and counterclockwise, before being recombined. In this case, the interference fringes arise due to an absolute rotation, and provide the most accurate measure of the angular velocity of the device. This was used by Michelson to measure the absolute rotation of the Earth. More recently, the Sagnac interference effect was cleverly used to measure time-reversal symmetry breaking in superconductors. ${ }^{3}$

Quantum mechanics opened the way for matter-wave interferometry. Electron interferometry is a powerful probe of interaction effects on low-energy phases of quantum matter, as demonstrated by numerous examples. Mach-Zehnder interferometers reveal Aharonov-Bohm oscillations and quantum Hall effect edge channels, ${ }^{4-8}$ and can probe exotic fractional quantum Hall states. ${ }^{9-11}$ Similarly, two-path MachZehnder interferometers can probe correlated states of quantum dots. ${ }^{12,13}$ Of particular interest to us are metallic carbon nanotubes. The Luttinger liquid behavior in these systems ${ }^{14-16}$ was partially verified through the observation of Fabry-Pérot interference in finite sections of the nanotube. ${ }^{17}$
The Fabry-Pérot interference should, in principle, allow the observation of spin-charge separation and determination of the interaction parameters of the Luttinger liquid. ${ }^{18}$ But the similar energy scales of the spin and charge modes' interference patterns have made such experimental observation challenging.

The most sensitive interferometer of all, however, the Sagnac interferometer, has not been seriously explored yet in the context of interacting electronic systems. In Ref. 19 we proposed that this interference naturally occurs in metallic armchair nanotube loops (Fig. 2). Instead of rotation, the Sagnac interference arises due to the band velocity difference between right- and left-moving electrons about each Dirac node. ${ }^{20}$ This velocity difference is present whenever the electronic Fermi surface is tuned away from the Dirac points at half-filling, as shown in Fig. 3(a). The operating principle of the electronic Sagnac effect has the same origin as the universal conductance fluctuations, and weak-localization effects in disordered two-dimensional electron gases. ${ }^{21-23}$ In nanotubes, it can also appear due to band scattering in a pair of impurities. ${ }^{24}$

Because the Sagnac effect involves electrons traversing the same path in two different directions, rather than repeating the same path as in Fabry-Pérot interference, the phase accumulation is extremely small. Therefore Sagnac interference exhibits large-period conductance fluctuations as a function of gate- and source-drain voltages, and is expected to persist to high temperatures in comparison to Fabry-Pérot interference, which is more sensitive to thermal dephasing. This interference mode should thus be able to reveal much more precise information about the unique state of interacting electrons in thin quantum wires.

Our goal in this manuscript is to thoroughly explore the range and robustness of the Sagnac interference mode, concentrating on armchair carbon nanotubes. The questions we will ask concern the amplitude of this interference mode as a function of the temperature, gate and source-drain voltages, and Luttinger parameter of the nanotube.

The paper is organized as follows. In Sec. II, as a warm up, we analyze the simpler case of Sagnac interference in a single channel of right- and left-moving electrons. In Sec. II A we introduce the model of a single channel with a lin- 
a. Mach-Zehnder
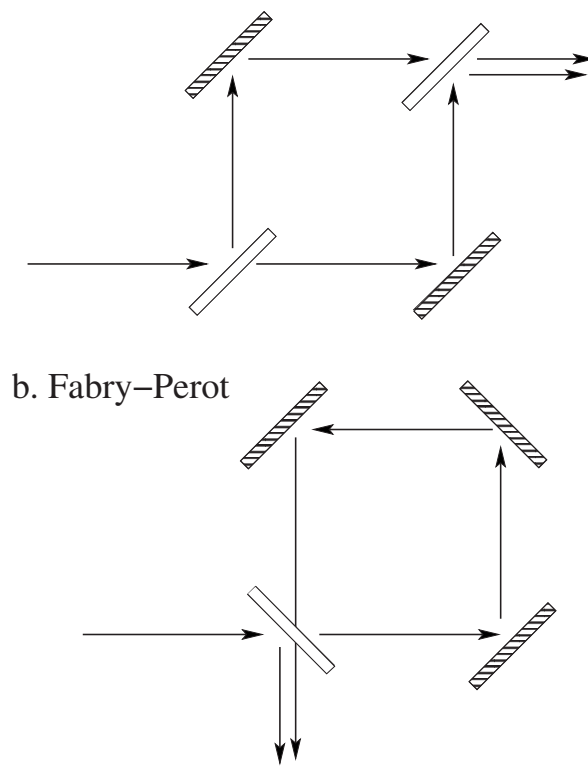

c. Sagnac

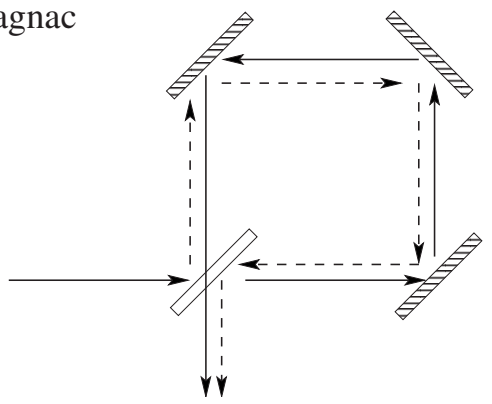

FIG. 1. (a) In a Mach-Zehnder interferometer the input beam is split into two beams which traverse independent paths before being recombined. (b) In a Fabry-Pérot interferometer a beam is split into a deflected ray, which is recombined at the output with a ray that traverses a loop. (c) The Sagnac interferometer splits the beam into a two beams which traverse the loop in two opposite orientations, and get recombined at the output. This allows a very sensitive measurement of the angular velocity of the interferometer, as it results in a different relative speed in the clockwise and counterclockwise rays. Clear rectangles represent beam splitters, and patterned rectangles represent mirrors.

earized spectrum, and the cross-loop tunneling which will give rise to the Sagnac interference. In Secs. II B and II C we set up the nonequilibrium perturbative calculation of the con-

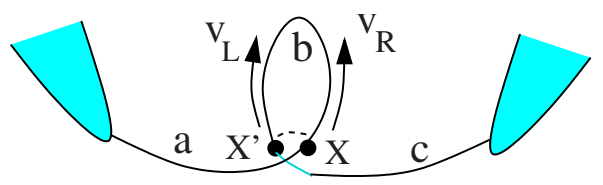

FIG. 2. (Color online) Schematic of a nanotube loop reported in Ref. 19. This geometry allows electrons to tunnel from the point $X$ on the loop to a distant point $X^{\prime}$ on the other end of the loop, and vice versa. We refer to this process as cross-loop tunneling. An electron entering from the left can traverse the loop moving right with velocity $v_{R}$, without scattering, or tunnel from $X$ to $X^{\prime}$ and traverse the loop moving left with velocity $v_{L}$. a.
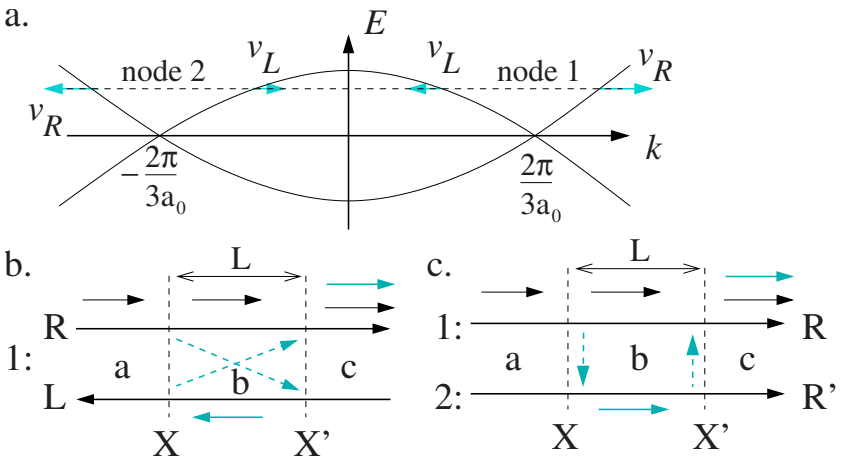

FIG. 3. (Color online) (a) The energy spectrum of an armchair nanotube. When the chemical potential is tuned away from the degeneracy points by a gate voltage, the left and right movers in each node will have different velocities, which leads to the Sagnac interference in the loop geometry. (b) This figure shows right- and leftmoving electrons in node 1 . The loop was unraveled in this figure, so the tunneling appears to be nonlocal, from point $X$ to point $X^{\prime}$, the two ends of the loop. The scattering shown in figure is from a right-moving electron from a given node at point $X$ to a left-moving electron, of the same node, at point $X^{\prime}$, and vice versa. This scattering gives rise to the Sagnac interference. (c) Sagnac interference can also arise without the loop geometry through internode tunneling, since right movers at node 2 have the same velocity as left movers at node 1 .

ductance in the presence of cross-loop tunneling, and in Secs. II D and II E we analyze the behavior of the oscillating conductance as a function of gate and bias voltages and temperature. In Sec. III we repeat the above steps for the physically relevant case of carbon nanotubes, including spin and node degeneracies in the calculation, and remark on the similarities and differences from the single-channel case. Finally we conclude with a discussion of the experimental implications of our calculations.

\section{SAGNAC INTERFERENCE IN A SINGLE CHANNEL}

As discussed in Sec. I, the Sagnac interference in the loop geometry is due the asymmetry between the velocities of the left- and right-moving electrons. To demonstrate this in the simplest form, we first study in this section a single channel with a single type of left and right movers. In a carbon nanotube, there will be four such channels due to spin and node degeneracies.

\section{A. Model}

We start with a single one-dimensional channel of electrons and a linearized spectrum, with different left- and rightmover velocities, and a density-density interaction. The Hamiltonian density for this system is:

$$
\mathcal{H}_{1 \mathrm{ch}}=-i \hbar v_{R} \psi_{R}^{\dagger} \partial_{x} \psi_{R}+i \hbar v_{L} \psi_{L}^{\dagger} \partial_{x} \psi_{L}+\lambda\left(\psi_{R}^{\dagger} \psi_{R}+\psi_{L}^{\dagger} \psi_{L}\right)^{2},
$$

where the operator $\psi_{R / L}^{\dagger}$ creates a right-/left-moving electron, with the velocity: 


$$
v_{R / L}=v_{F} \pm u
$$

The scattering we are interested in is the one which takes a right-moving electron at one side of the loop, point $X$ is Fig. 2 , and scatters it to a left-moving electron at the other side of the loop, point $X^{\prime}$, and vice versa. This process has been dubbed cross-loop scattering in Ref. 19. If we choose our coordinate along the loop such that the point $X$ corresponds to $x=0$ and the point $X^{\prime}$ corresponds to $x=L$, then this scattering process is described by the Hamiltonian

$$
H_{\mathrm{bs}}=\Gamma_{1} \psi_{R}^{\dagger}(0) \psi_{L}(L)+\text { H.c. }+\Gamma_{2} \psi_{L}^{\dagger}(0) \psi_{R}(L)+\text { H.c. }
$$

In the presence of the quartic density-density interactions in the Hamiltonian, Eq. (1), it is useful to use the standard bosonization procedure, since the Hamiltonian is quadratic in terms of the bosonic fields. The electron fields are bosonized as follows:

$$
\psi_{R / L} \sim e^{i(\phi \pm \theta)},
$$

where $\theta$ and $\phi$ are bosonic fields that satisfy the commutation relations $\left[\theta(x), \phi\left(x^{\prime}\right)\right]=i(\pi / 2) \operatorname{sgn}\left(x-x^{\prime}\right)$; also, the total density and the current density are given by $\frac{1}{\pi} \nabla \theta=\rho_{R}+\rho_{L}$, and $\frac{1}{\pi} \nabla \phi=\rho_{R}-\rho_{L}$, respectively. The Hamiltonian in terms of the bosonic fields becomes

$$
H_{1 \mathrm{ch}}=\frac{\hbar v}{2 \pi} \int d x\left[\frac{1}{g}(\nabla \theta)^{2}+g(\nabla \phi)^{2}+2 \frac{u}{v} \nabla \theta \nabla \phi\right],
$$

where $g=\left(1+\frac{2 \lambda}{\pi \hbar v_{F}}\right)^{-1 / 2}$ is the Luttinger interaction parameter and $v=v_{F} / g$. This is the familiar Hamiltonian of a onedimensional (1D) interacting electron system, with the addition of the $u$ term which gives left and right-moving particles different velocities. Indeed, this Hamiltonian can be easily diagonalized and the left and right velocities turn out to be for a general value of the interaction parameter $g$ :

$$
v_{R / L}=v \pm u=\frac{v_{F}}{g} \pm u .
$$

Our goal is to calculate the effects of the Sagnac interference as seen in the conductance as a function of the applied bias and gate voltages, and as a function of temperature. Due to the applied voltages the system is not in equilibrium, and we must turn to the Keldysh nonequilibrium formalism. ${ }^{25,26}$ Below we carry out this analysis first for the simplified electron gas with the scattering Hamiltonian $H_{\mathrm{bs}}$, Eq. (3), as a perturbation.

\section{B. Nonequilibrium correlation functions and conductance}

The response of the loop to a bias source-drain voltage can be analyzed using the nonequilibrium Keldysh formalism. Following Ref. 18, we assume that in the distant past, before turning on the backscattering, the left- and rightmoving electrons separately had well defined thermal distributions set by separate chemical potentials. The density matrix corresponding to this initial distribution at temperature $T=1 / \beta$ is

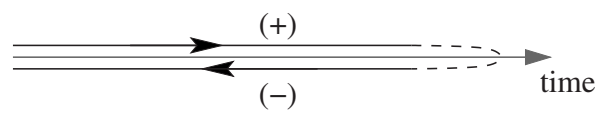

FIG. 4. The Keldysh contour used in the nonequilibrium calculation. The Keldysh time-ordering operator $T_{K}$ orders operators along the contour, so fields on the $(+)$ branch are always at an earlier time than fields on the $(-)$ branch.

$$
\hat{\rho}_{V}=\frac{1}{Z_{V}} e^{-\beta \hat{H}_{V}}
$$

with $Z_{V}=\operatorname{Tr}\left[e^{-\beta \hat{H}_{V}}\right]$ and the Hamiltonian which takes into account the applied voltages is

$$
\begin{aligned}
H_{V} & =H_{1 \mathrm{ch}}-e \frac{V_{\mathrm{sd}}}{2}\left(N_{R}-N_{L}\right)-\alpha e V_{g}\left(N_{R}+N_{L}\right) \\
& =H_{1 \mathrm{ch}}-e \frac{V_{\mathrm{sd}}}{2} \int d x \frac{\nabla \phi}{\pi}-\alpha e V_{g} \int d x \frac{\nabla \theta}{\pi} .
\end{aligned}
$$

The gate voltage, $V_{g}$, simply couples to the total charge density, with $\alpha$ being a geometrical factor of the system, while the source-drain voltage, $V_{\mathrm{sd}}$, induces the imbalance in the chemical potentials of the left and right movers.

As explained in Ref. 18, both $V_{\text {sd }}$ and $V_{g}$ can be eliminated from the unperturbed action by an appropriate unitary transformation, which is equivalent to shifting the bosonic fields by a function of space and time; this is easy to see if one writes down the Lagrangian including the voltages. ${ }^{19}$ The equivalent shifts for the case at hand are

$$
\begin{gathered}
\theta \rightarrow \theta+\frac{\alpha g^{2} e V_{g}}{\hbar v_{F}} \frac{1}{1-g^{2} u^{2} / v_{F}^{2}} x-\frac{e V_{\mathrm{sd}}}{2 \hbar} t, \\
\phi \rightarrow \phi-\frac{\alpha g^{2} e V_{g}}{\hbar v_{F}} \frac{u / v_{F}}{1-g^{2} u^{2} / v_{F}^{2}} x .
\end{gathered}
$$

These shifts remove the voltages from the Hamiltonian $H_{V}$ and therefore all the correlations to appear in the calculation will be equilibrium correlation functions with respect to $H_{1 \mathrm{ch}}$. The dependence on the applied voltages now appears in the scattering Hamiltonian, $H_{\mathrm{bs}}$, due to the shifted bosonic fields.

Let us now focus our attention at the charge current, which in the bosonic language is $\hat{I}=(e / \pi) \partial_{t} \theta$. After performing the unitary transformation described above we can write the formal expression for the expectation value of the current in the usual interaction picture: ${ }^{18}$

$$
\langle I\rangle=I_{0}+\frac{1}{Z_{V=0}} \operatorname{Tr}\left(e^{-\beta H_{1 \mathrm{ch}}} \hat{T}_{K}\left\{\hat{I}_{K}(x, t) e^{-i \int_{\mathcal{C}} d t^{\prime} H_{\mathrm{bs}}^{\prime}\left(t^{\prime}\right)}\right\}\right) .
$$

$\hat{T}_{K}$ is the time-ordering operator along the Keldysh contour shown in Fig. 4 , and $\hat{I}_{K}(x, t)$ is the symmetrized current operator with respect to the two branches of the contour. The current $I_{0}=e^{2} V_{\mathrm{sd}} / h$ is the ideal current that would flow in the absence of backscattering in a completely transmitting channel, and it explicitly appears due to the shift of the $\theta$ field. The Hamiltonian $H_{\mathrm{bs}}^{\prime}$ denotes the scattering Hamiltonian $H_{\mathrm{bs}}$ 
with the properly shifted bosonic fields. The expression for the current can be expanded in powers of $H_{\mathrm{bs}}^{\prime}$, and all the correlation functions to appear in this expansion are equilibrium correlation functions at temperature $1 / \beta$. If we denote by $\theta^{+}$and $\theta^{-}$the fields on the forward branch and backward branch of the Keldysh contour, respectively, then time ordering along the contour means that $\theta^{+} \theta^{+}$correlations have the usual time ordering, $\theta^{-} \theta^{-}$are antitime ordered, and $\theta^{+}(t)$ is always earlier in time that $\theta^{-}\left(t^{\prime}\right)$. The same applies for all the fields.

It is useful to apply a Keldysh rotation to the fields, $\theta^{ \pm}=\theta \pm \frac{i}{2} \tilde{\theta}$, and similarly for $\phi$. The correlation function $\left\langle T_{K} \tilde{\theta}(t) \widetilde{\theta}\left(t^{\prime}\right)\right\rangle$ vanishes by construction, and we define

$$
\begin{aligned}
& C^{\theta}\left(x, t ; x^{\prime}, t^{\prime}\right)=\left\langle T_{K} \theta(x, t) \theta\left(x^{\prime}, t^{\prime}\right)\right\rangle=\frac{1}{2}\left\langle\left\{\hat{\theta}(x, t), \hat{\theta}\left(x^{\prime}, t^{\prime}\right)\right\}\right\rangle \\
& R^{\theta}\left(x, t ; x^{\prime}, t^{\prime}\right)=\left\langle T_{K} \theta(x, t) \tilde{\theta}\left(x^{\prime}, t^{\prime}\right)\right\rangle \\
&=-i \Theta\left(t-t^{\prime}\right)\left\langle\left[\hat{\theta}(x, t), \hat{\theta}\left(x^{\prime}, t^{\prime}\right)\right]\right\rangle
\end{aligned}
$$

and similarly for the $\phi_{j}$ fields, and for the mixed correlations

$$
\begin{aligned}
& C^{\theta \phi}\left(x, t ; x^{\prime}, t^{\prime}\right)=\left\langle T_{K} \theta(x, t) \phi\left(x^{\prime}, t^{\prime}\right)\right\rangle=\frac{1}{2}\left\langle\left\{\hat{\theta}(x, t), \hat{\phi}\left(x^{\prime}, t^{\prime}\right)\right\}\right\rangle \\
& R^{\theta \phi}\left(x, t ; x^{\prime}, t^{\prime}\right)=\left\langle T_{K} \theta(x, t) \widetilde{\phi}\left(x^{\prime}, t^{\prime}\right)\right\rangle \\
&=-i \Theta\left(t-t^{\prime}\right)\left\langle\left[\hat{\theta}(x, t), \hat{\phi}\left(x^{\prime}, t^{\prime}\right)\right]\right\rangle,
\end{aligned}
$$

where operators with a hat are simply the time dependent operators with no time ordering. As explained above, these correlation function are to be evaluated in equilibrium, and therefore are easily explicitly calculated (Appendix B). Due to translational invariance in time and space, these correlations are functions of $x-x^{\prime}$ and $t-t^{\prime}$, for example:

$$
\begin{aligned}
C^{\theta \phi}(x, t) \equiv & C^{\theta \phi}(x, t ; 0,0)=\frac{1}{4}\left\{\log \left[v_{R} \sinh \left(\frac{\left(v_{R} t-x\right) \pi}{\beta v_{R}}\right)\right]\right. \\
& \left.-\log \left[v_{L} \sinh \left(\frac{\left(v_{L} t+x\right) \pi}{\beta v_{L}}\right)\right]\right\} .
\end{aligned}
$$

\section{Perturbation theory}

The Sagnac interference fringes occur already with weak backscattering at the base of the loop, and can be deduced from a perturbation analysis of the tunneling Hamiltonian [Eq. (3)]. As outlined above, to calculate the current, $I_{1 \mathrm{ch}}$ $=\left\langle\frac{e}{\pi} \partial_{t} \theta\right\rangle$, we absorb the gate and bias voltages, $V_{g}$ and $V_{\text {sd }}$, respectively, in the shifts in Eq. (9), which allow us to move the voltages from the unperturbed Hamiltonian $H_{1 \mathrm{ch}}$ to the backscattering perturbation, $H_{\mathrm{bs}}$. Then, we expand the formal expression we found for the current using the Keldysh technique [Eq. (10)] in powers of $H_{\mathrm{bs}}$, and use Wick's theorem to evaluate the resulting contributions.

To lowest nontrivial order, which is second order in $H_{\mathrm{bs}}$, we obtain after a lengthy calculation:

$$
I_{1 \mathrm{ch}}=\frac{e^{2} V_{\mathrm{sd}}}{h}+I_{\mathrm{co}}+I_{\text {inco }} .
$$

The first term is simply the current that would flow through the system in the absence of backscattering. The coherent current, $I_{\text {co }}$, oscillates with the gate voltages $V_{g}$, and is given by:

$$
\begin{aligned}
I_{\mathrm{co}}= & c \Gamma_{1} \Gamma_{2} \cos \left[\frac{2 u g^{2} L \alpha}{\hbar^{2} v_{F}^{2}\left(1-g^{2} u^{2} / v_{F}^{2}\right)} V_{g}\right] \\
& \times \int d t \sin \left(\frac{e V_{\mathrm{sd}}}{\hbar} t\right) e^{-C_{\mathrm{co}}(L, t)} \sin \left[R_{\mathrm{co}}(L, t)\right],
\end{aligned}
$$

where $c$ is a constant of order unity, and we assume that $\Gamma_{i}$ is real for simplicity. The incoherent current, $I_{\text {inco }}$, is independent of the gate voltage, and is given by:

$$
\begin{aligned}
I_{\text {inco }}= & c\left\{\Gamma_{1}^{2} \sum_{\eta= \pm} \int d t \sin \left(\frac{e V_{\text {sd }}}{\hbar} t\right) e^{-C_{\text {inco }}^{\eta}(L, t)} \sin \left[R_{\text {inco }}(L, t)\right]\right\} \\
& +c\left(\Gamma_{1} \rightarrow \Gamma_{2}, L \rightarrow-L\right) .
\end{aligned}
$$

The functions $C_{\mathrm{co}}, C_{\mathrm{inco}}^{ \pm}, R_{\mathrm{co}}$, and $R_{\text {inco }}$ are complicated combinations of the correlation functions defined in Sec. II B and are given explicitly in Appendix B. These functions do not simplify, partly due to the fact that the correlation functions in this problem are not symmetric under $x \rightarrow-x$ since left and right movers have different velocities.

\section{Voltage dependence of the single-mode Sagnac interference}

The voltage current characteristics given in Eqs. (14)-(16) can be evaluated numerically to obtain the voltage and temperature dependence of the single-mode Sagnac interference. The period of the interference as a function of the gate voltage $\left(I_{\mathrm{co}}\right)$ is easily observed to be (for small $\left.u / v_{F}\right)$ :

$$
\Delta V_{g}^{\text {Sagnac }} \approx \frac{v_{F}}{u} \frac{\pi \hbar^{2} v_{F}}{\alpha g^{2} L}=\frac{v_{F}}{u} \Delta V_{g}^{\mathrm{FP}},
$$

where $\Delta V_{g}^{\mathrm{FP}}$ is the period in gate voltage for Fabry-Pérot interference. Fabry-Pérot interference occurs whenever part of the wave's trajectory can be repeated. Since the Sagnac interference involves traversing the same path in two different directions, the phase difference accumulated in the process is much smaller than the difference incurred by repeating part of the path, and therefore the period of the Sagnac interference is much larger than the period of the Fabry-Pérot interference. Such large-period oscillations have been experimentally observed in carbon nanotubes, in the loop geometry, as reported in Ref. 19, in addition to the shorter period Fabry-Pérot oscillations.

For a given gate voltage, both the coherent and incoherent parts of the current oscillate with the bias voltage $V_{\text {sd }}$. This oscillation is due to the fact that in the presence of bias voltage, the Fermi energy of the left- and right-moving electrons is different by $V_{\text {sd }}$, and hence their Fermi wave vectors are different also and they would acquire different phases traversing the loop. This oscillation will be present even for no velocity detuning, $u=0$. When the detuning is finite, $u$ 


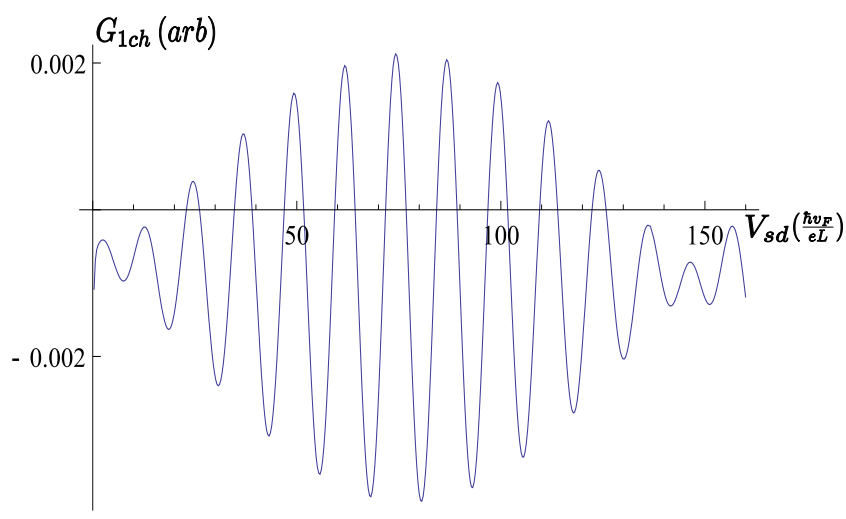

FIG. 5. (Color online) Differential conductance oscillations of a single channel of fermions, as a function of bias voltage $V_{\text {sd }}$, for velocity detuning $u / v_{F}=0.1$ and interaction strength $g=0.5$. The beating is due to the only two voltage oscillation frequencies in the problem, $\Omega_{1}=\frac{e L}{\hbar(v+u)}$ and $\Omega_{2}=\frac{e L}{\hbar(v-u)}$, where $v=v_{F} / g$. The voltage is in units of $\hbar v_{F} / e L$. The shorter voltage oscillation period is $\Delta V_{\mathrm{sd}}$ $=2 \pi\left(\frac{\Omega_{1}+\Omega_{2}}{2}\right)^{-1} \approx 12.5 \hbar v_{F} / e L$, and the large oscillation period is $\Delta V_{\mathrm{sd}}=2 \pi\left(\frac{\Omega_{1}-\Omega_{2}}{2}\right)^{-1} \approx 250 \hbar v_{F} / e L$.

$\neq 0$, the differential conductance $G_{1 \mathrm{ch}}=\partial I_{1 \mathrm{ch}} / \partial V_{\text {sd }}$ will show a beating pattern due to the two different left and rightmoving excitation velocities. Here we are only considering the Sagnac oscillations arising from the cross-loop tunneling [Eq. (3)]. Figure 5 shows the oscillations of the differential conductance at a fixed gate voltage. For noninteracting electrons, the beating pattern corresponds to the addition of two harmonics with two different frequencies in voltage, $\sin \left(\Omega_{R} V_{\text {sd }}\right)$ and $\sin \left(\Omega_{L} V_{\text {sd }}\right)$, with $\Omega_{R / L}=\frac{e L}{\hbar v_{R / L}}$ and $v_{R / L}$ $=v_{F} \pm u$. The beating pattern will then display fast oscillations with voltage period $\Delta V_{\mathrm{sd}}^{\mathrm{fast}}=2 \pi\left(\frac{\left|\Omega_{R}+\Omega_{L}\right|}{2}\right)^{-1}$, and slow voltage oscillations with period $\Delta V_{\mathrm{sd}}^{\text {slow }}=2 \pi\left(\frac{\Omega_{R}-\Omega_{L}}{2}\right)^{-1}$. For interacting fermions, $g \neq 1$, the oscillations will not be simple harmonic oscillations. Still, the periods will be evident and will have the same functional form, in terms of $v_{R / L}$, as the frequencies in the noninteracting case. The periods $\Delta V_{\mathrm{sd}}^{\mathrm{fast}}$ and $\Delta V_{\text {sd }}^{\text {slow }}$ do depend on $g$ through velocities $v_{L}$ and $v_{R}$, $v_{R / L}=\frac{v_{F}}{g} \pm u$ [Eq. (6)].

These oscillation, generally, lie atop a power-law behavior of the differential conductance as a function of $V_{\text {sd }}$, as expected from the known behavior of the conductance in the presence of impurity backscattering. ${ }^{27}$ For backscattering from an impurity in a Luttinger liquid, the backscattered current, for low temperature, behaves as $I \propto V_{\mathrm{sd}}^{2 g-1}$. We chose to plot the Sagnac oscillations as a function of $V_{\text {sd }}$ (Fig. 5) for the interaction parameter $g=0.5$ since for that value the corresponding power law would be $I \propto V_{\text {sd }}^{0}$, and the contribution of such a power law to the differential conductance would vanish, making the oscillation atop this power law more visible.

\section{E. Temperature dependence of the single-mode Sagnac interference}

Next we consider the temperature dependence of the gatevoltage driven oscillations in the coherent part of the current.
As argued in Ref. 19, the large-period Sagnac oscillations are expected to be observed at much higher temperature than the shorter period Fabry-Pérot oscillations. This difference in temperature behavior can be easily understood by examining the phase giving rise to the interference in both cases. In the Fabry-Pérot case for a loop, the lowest-order interference is between a beam of electrons which is not scattered, and a beam of electrons which, due to scattering at the base of the loop, does a roundtrip between the two scattering points. The phase difference between these two beams at energy $E$ is $\Delta \phi_{\mathrm{FP}}=k_{R} L=\frac{1}{v_{R}} L \frac{E}{\hbar}$. Finite temperature effectively causes uncertainty of order $T$ in the energy $E$, and the interference pattern will be washed out when the uncertainty of the phase $\Delta \phi_{\mathrm{FP}}$ is of order $2 \pi$, which happens at a temperature $T_{\mathrm{FP}}$ $=\frac{2 \pi \hbar}{L} v_{R}$.

In the Sagnac case, the interference is between a beam that traverses the loop moving left and one which traverses the loop moving right. The phase difference between these two beams at energy $E$ is $\Delta \phi_{\mathrm{SAG}}=k_{L} L-k_{R} L=\left(\frac{1}{v_{L}}-\frac{1}{v_{R}}\right) L \frac{E}{\hbar}$, and this interference will be washed out at temperature $T_{\mathrm{SAG}}$ $=\frac{2 \pi \hbar}{L}\left(\frac{1}{v_{L}}-\frac{1}{v_{R}}\right)^{-1}$. For noninteracting electrons the right and left-moving velocities are $v_{R / L}=v_{F} \pm u$. Thus to lowest order in $u / v_{F}$, the highest temperatures for observing interference according to the argument above are

$$
T_{\mathrm{FP}} \approx \frac{\pi \hbar v_{F}}{L}, \quad T_{\mathrm{SAG}} \approx \frac{\pi \hbar v_{F}}{L} \frac{v_{F}}{u}=T_{\mathrm{FP}} \cdot \frac{v_{F}}{u} .
$$

For noninteracting electrons, we expect the Sagnac interference to survive to a temperature higher by a factor of $v_{F} / u$ than the corresponding Fabry-Pérot temperature. We will show through explicit calculation that this is indeed true for the noninteracting case. For interacting electrons, we will see that $T_{\mathrm{SAG}}$ will still be considerably larger than $T_{\mathrm{FP}}$, but their ratio is less than the dramatic $v_{F} / u$ ratio.

To explore the Sagnac temperature range, we evaluate the amplitude of the coherent oscillations (the oscillations in $V_{g}$ ) as a function of temperature, for different interaction parameters $g$ and different ratios of $u / v_{F}$. For noninteracting electrons, $g=1$, we find that the Sagnac oscillations indeed survive up to a high temperature, which is a factor of $v_{F} / u$ higher than the corresponding Fabry-Pérot oscillations. Figure 6 plots the oscillation amplitude as a function of temperature, normalized by its zero-temperature value, and for different values of $u / v_{F}$. The functional dependence on temperature is given approximately by:

$$
\frac{G_{\mathrm{co}}(T)}{G_{\mathrm{co}}(T=0)}=\left(\frac{2 \pi k_{B} L T}{\hbar v_{F}}\right)\left(\frac{u}{v_{F}}\right) \frac{1}{\sinh \left(2 \pi k_{B} T \frac{L}{\hbar v_{F}} \frac{u}{v_{F}}\right)} .
$$

This result is similar to the exact form of the temperature dependence of the Fabry-Pérot interference amplitude, ${ }^{28}$ with the only difference being the factor of $u / v_{F}$. Therefore, the Sagnac oscillations of noninteracting electrons indeed survive up to temperatures which are a factor of $v_{F} / u$ larger than the Fabry-Pérot oscillations.

For interacting electrons, $g \neq 1$, the Sagnac interference still survives up to temperatures significantly higher than the corresponding Fabry-Pérot temperature scales, but the en- 


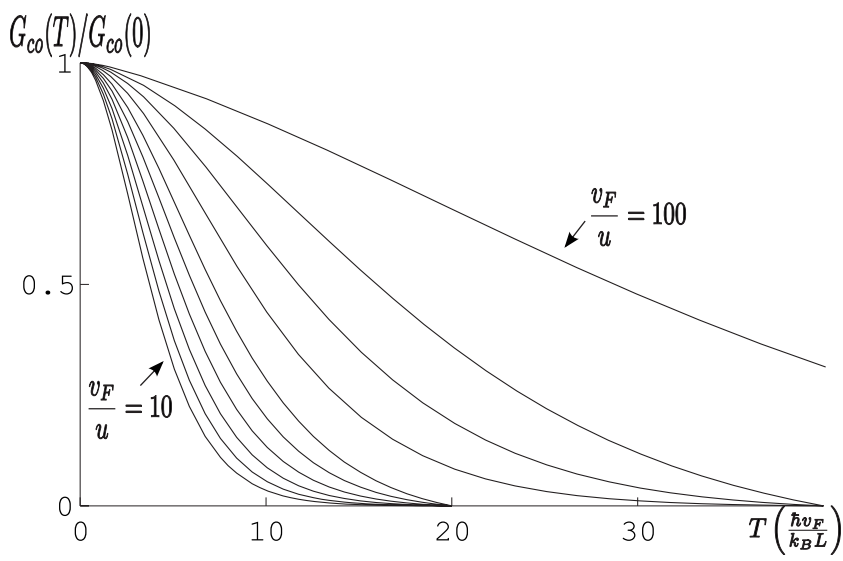

FIG. 6. Coherent Sagnac oscillation amplitude vs temperature for different values of $\left(v_{F} / u\right)$, for noninteracting electrons $(g=1)$. The slowest decaying plot corresponds to $v_{F} / u=100$, and the fastest decaying plot corresponds to $v_{F} / u=10$. Temperature is given in units of $\hbar v_{F} / k_{B} L$.

hancement is suppressed compared to that of noninteracting electrons. Figure 7 shows the Sagnac temperature scale $T^{*}$ vs $u / v_{F}$ for three different values of the interaction parameter $g$, where we define $T^{*}$ to be the temperature at which the amplitude of the oscillations reaches $e^{-1}$ of its amplitude at zero temperature. For noninteracting electrons $T^{*}$ is strongly dependent on the ratio $u / v_{F}$ as discussed above. For the interaction parameter values $g=0.5$ and $g=0.25$ (dashed lines), the temperature $T^{*}$ is only weakly dependent on the ratio $u / v_{F}$. As an example for the resulting enhancement of the Sagnac compared to the Fabry-Pérot interference, consider

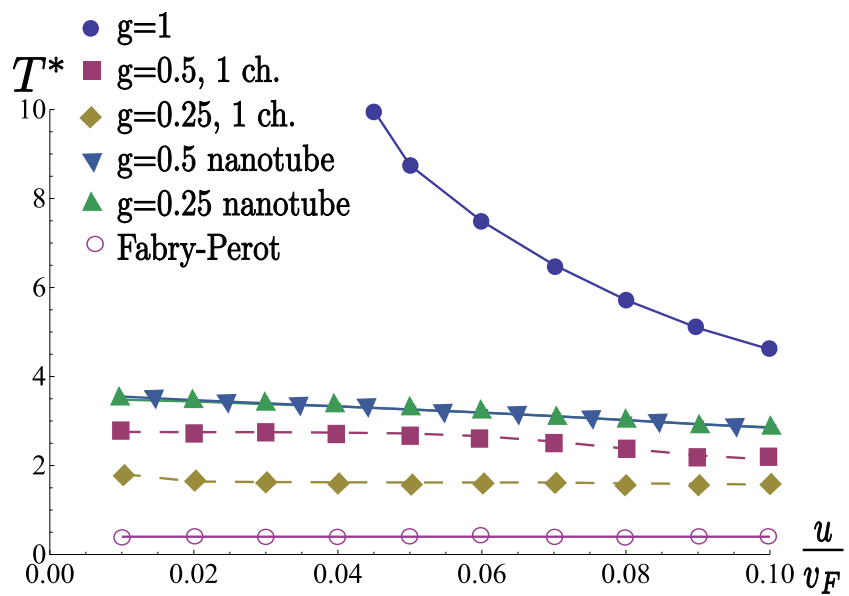

FIG. 7. (Color online) $T^{*}$ vs $u / v_{F}$, where $T^{*}$ is the temperature at which the coherent differential conductance (the part of the conductance which oscillates with gate voltage) reaches $e^{-1}$ of its zerotemperature value. For a noninteracting system, $g=1$, the singlechannel case gives the same temperature dependence as the case with spin and node degeneracies, $T^{*} \propto v_{F} / u$. The single-channel temperature dependence is given for $g=0.5$ (squares, dashed), and $g=0.25$ (diamonds, dashed). The carbon nanotube temperature dependence is given for $g=0.5$ (triangles), and $g=0.25$ (inverted triangles). Temperature is given in units of $\hbar v_{F} / k_{B} L$. For reference, the $T^{*}$ corresponding to the $g=1$ Fabry-Pérot oscillations is also plotted. $g=0.25$, where the $T^{*}$ temperature scale for the Sagnac oscillations is roughly $1.6 \hbar v_{F} / k_{B} L$, a factor of 4 enhancement over $T^{*}$ of the noninteracting Fabry-Pérot oscillations which is $0.42 \hbar v_{F} / k_{B} L$, despite the suppression of the Sagnac $T^{*}$ due to interactions. As can be seen in the figure, for $g=0.5$ the enhancement is about 7 . While it is difficult to extract the analytic dependence of the temperature on the interaction parameter, one can repeat our calculation for any value of $g$.

\section{INTERFERENCE IN NANOTUBES}

Equipped with our understanding of the single-channel Sagnac interference, we can now consider the likely physical system where it may be observed: a metallic carbon nanotube with four different Dirac nodes. We now add the spin and node degeneracies of a carbon nanotube, and examine their effect on the Sagnac interference pattern voltage and temperature dependence.

\section{A. Model}

The energy spectrum of a carbon nanotube is shown in Fig. 3(a). This spectrum is usually linearized around the Fermi surface, which yields four chiral modes, two left moving and two right moving (not including spin), with linear dispersion. These modes can be bosonized and treated within the Luttinger Liquid theory framework, as we have done in the single-channel case in Sec. II. All these modes are usually assumed to have the same velocity, the Fermi velocity $v_{F}$. For the purposes of this paper, it is important to notice that when the Fermi surface is away from the degeneracy points where the upper and lower bands meet, linearizing the spectrum actually gives two different velocities which we shall note $v_{ \pm}=v_{F} \pm u$. The linearized Hamiltonian density is, then

$$
\begin{aligned}
\mathcal{H}_{4 \mathrm{ch}}= & i \sum_{a=1}^{2} \sum_{\sigma=\uparrow, \downarrow}\left(v_{R a} \psi_{R a \sigma}^{\dagger} \partial_{x} \psi_{R a \sigma}-v_{L a} \psi_{L a \sigma}^{\dagger} \partial_{x} \psi_{R a \sigma}\right) \\
& +\lambda\left[\sum_{a=1}^{2} \sum_{\sigma=\uparrow, \downarrow}\left(\psi_{R a \sigma}^{\dagger} \psi_{R a \sigma}+\psi_{L a \sigma}^{\dagger} \psi_{L a \sigma}\right)\right]^{2},
\end{aligned}
$$

where $\psi_{R / L a \alpha}$ stands for a right-/left-moving electron at node $a$ with spin $\sigma$, and we added a total charge-density interaction term. The velocities that appear in the Hamiltonian are

$$
\begin{gathered}
v_{R / L 1 \sigma}=v_{F} \pm u=v_{ \pm} \\
v_{R / L 2 \sigma}=v_{F} \mp u=v_{\mp} .
\end{gathered}
$$

Thus for $u>0$, at node 1 right movers are faster than left movers, while at node 2 the opposite is true. Now, the nonlinearity of the electronic spectrum in a carbon nanotube needs to be taken into account when considering the velocity difference, $u$; it depends on the detuning of the chemical potential away from the degeneracy points.

The scattering process we are interested in is very similar to the one we had in the single-channel case. We need to consider a term that scatters a right mover at one end of the 
loop to a left mover at the other end of the loop, conserving spin and node quantum numbers,

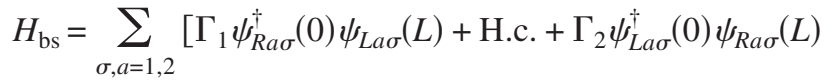

$$
\begin{aligned}
& + \text { H.c.]. }
\end{aligned}
$$

Next we bosonize the electron field operators in the nanotube. The slowly oscillating parts can be written as

$$
\psi_{R / L a \sigma} \sim e^{i\left(\phi_{a \sigma} \pm \theta_{a \sigma}\right)},
$$

where $\theta_{a \sigma}$ and $\phi_{a \sigma}$ are bosonic fields that satisfy the commutation relations $\left[\theta_{a \sigma}(x), \phi_{a^{\prime} \sigma^{\prime}}\left(x^{\prime}\right)\right]=i(\pi / 2) \delta_{a, a^{\prime}} \delta_{\sigma, \sigma^{\prime}} \operatorname{sgn}(x$ $\left.-x^{\prime}\right)$. The Hamiltonian in terms of the bosonic fields is ${ }^{19}$

$$
\begin{aligned}
H_{4 \mathrm{ch}}= & \frac{\hbar v_{F}}{2 \pi} \sum_{\sigma, a=1,2} \int d x\left[\left(\nabla \theta_{a \sigma}\right)^{2}\right. \\
& \left.+\left(\nabla \phi_{a \sigma}\right)^{2}+(-1)^{a+1} 2 \frac{u}{v_{F}} \nabla \phi_{a \sigma} \nabla \theta_{a \sigma}\right] \\
& +\int d x \lambda\left(\sum_{\sigma, a=1,2} \frac{1}{\pi} \nabla \theta_{a}^{\sigma}\right)^{2} .
\end{aligned}
$$

If the velocities of all branches of the spectrum were equal, i.e., $u=0$, then the Hamiltonian $H_{B}$ would be diagonalized by the spin and node symmetric and antisymmetric combinations of the $\theta$ 's and $\phi$ 's. ${ }^{15}$ By diagonalizing we mean a linear mapping of the $\phi$ and $\theta$ fields such that the Hamiltonian takes the form of four independent channels, each resembling of $H_{1 \mathrm{ch}}$ [Eq. (5)]. When $u \neq 0$, there still exists a local transformation $\theta_{a \sigma}=\sum_{j=1 . .4}\left(A_{a \sigma}^{j} \theta_{j}+B_{a \sigma}^{j} \phi_{j}\right)$ that diagonalizes the Hamiltonian, but it is a more complicated combination of the fields that depends on $u$ and $\lambda$, and mixes the $\theta$ and $\phi$ fields, which makes the conductance calculation quite cumbersome. While the details of this transformation are given in Appendix A, the diagonal Hamiltonian is

$$
\begin{aligned}
H_{4 \mathrm{ch}}= & \sum_{j=3,4} \frac{\hbar v_{j}}{2 \pi} \int d x\left[\frac{1}{g_{j}}\left(\nabla \theta_{j}\right)^{2}+g_{j}\left(\nabla \phi_{j}\right)^{2}\right] \\
& +\sum_{j=1,2} \frac{\hbar v_{F}}{2 \pi} \int d x\left[\left(\nabla \theta_{j}\right)^{2}+\left(\nabla \phi_{j}\right)^{2}\right. \\
& \left.+(-1)^{j+1} 2 \frac{u}{v_{F}} \nabla \phi_{j} \nabla \theta_{j}\right] .
\end{aligned}
$$

The fields $\theta_{1 / 2}$ and $\phi_{1 / 2}$ are the spin antisymmetric combinations of $\theta_{1 / 2 \sigma}$ and $\phi_{1 / 2 \sigma}$, respectively. Since the interaction term in Eq. (20) involves only the spin-symmetric combinations, the spin antisymmetric combinations are untouched and still have the left- and right-moving velocities as in Eq. (21). On the other hand, the fields $\theta_{3 / 4}$ and $\phi_{3 / 4}$ are not simply the remaining symmetric combination and mix the remaining $\theta$ 's and $\phi$ 's. These fields have the same left- and right-moving velocity, which is

$$
v_{3 / 4}=\frac{v_{F}}{\sqrt{2}} \sqrt{1+\frac{1}{g^{2}}+2 \frac{u^{2}}{v_{F}^{2}} \pm \sqrt{\left(1-\frac{1}{g^{2}}\right)^{2}+8 \frac{u^{2}}{v_{F}^{2}}\left(1+\frac{1}{g^{2}}\right)}},
$$

where $g=\left(1+\frac{8 \lambda}{\pi \hbar v_{F}}\right)^{-1 / 2}$ is the Luttinger parameter. ${ }^{29}$

Fortunately, for the region of parameters which is of interest, namely strong interactions, $g \leq 0.5$ and $u / v_{F} \leq 0.1$, the exact change of basis required to diagonalize the spinsymmetric part of the Hamiltonian is very close to the usual node symmetric or antisymmetric basis. This can be explicitly seen, for example, from the velocities of these modes. For this entire range of parameters, the velocities of the diagonal fields, given by Eq. (26), are at most $1 \%$ different from the values we expect for the left-right symmetric system, which are $v_{F} / g$ and $v_{F}$. Due to the strong interactions in this spin-symmetric sector, the velocity asymmetry is unimportant, and it is for this reason that we choose to still use the node symmetric-antisymmetric basis and treat these fields as the diagonal ones. In Appendix A we elaborate on and justify this approximation. Note that the velocity asymmetry is still apparent in the noninteracting spin antisymmetric modes labeled by $j=1$ and $j=2$ in Eq. (25).

\section{B. Perturbation theory}

Using the diagonal form of the Hamiltonian with the above approximation, we proceed to calculate the current, $I$ $=(e / \pi)\left\langle\Sigma_{a \sigma} \partial_{t} \theta_{a \sigma}\right\rangle$, as in Sec. II. The applied voltages now couple to the total density and total number of left movers and right movers:

$$
\begin{aligned}
H_{V} & =H_{4 \mathrm{ch}}-e \frac{V_{\mathrm{sd}}}{2}\left(N_{R}-N_{L}\right)-\alpha e V_{g}\left(N_{R}+N_{L}\right) \\
& =H_{4 \mathrm{ch}}-e \frac{V_{\mathrm{sd}}}{2} \int d x \sum_{\sigma, a} \frac{\nabla \phi_{a \sigma}}{\pi}-e V_{g} \int d x \sum_{\sigma, a} \frac{\nabla \theta_{a \sigma}}{\pi} .
\end{aligned}
$$

The external voltages can be removed from the Hamiltonian by the appropriate shift of the bosonic fields:

$$
\begin{gathered}
\theta_{a \sigma} \rightarrow \theta_{a \sigma}+\frac{\alpha g^{2} e V_{g}}{\hbar v_{F}} \frac{1}{1-g^{2} u^{2} / v_{F}^{2}} x-\frac{e V_{\mathrm{sd}}}{2 \hbar} t, \\
\phi_{a \sigma} \rightarrow \phi_{a \sigma}+(-1)^{a} \frac{\alpha g^{2} e V_{g}}{\hbar v_{F}} \frac{u / v_{F}}{1-g^{2} u^{2} / v_{F}^{2}} x .
\end{gathered}
$$

Again we use the Keldysh contour to write the formal expression for the current, as in Eq. (10), and expand it to lowest order in the appropriate $H_{\mathrm{bs}}^{\prime}$ which contains the voltage dependence due to the shifts of the fields. The approximation we made above, namely that it is the node symmetric or antisymmetric combination which diagonalize the Hamiltonian, allows us to write the current in a very similar form to the single-channel case:

$$
I_{4 \mathrm{ch}}=4 \frac{e^{2} V_{\mathrm{sd}}}{h}+\tilde{I}_{\mathrm{co}}+\tilde{I}_{\text {inco }} .
$$


The first term on the right-hand side of Eq. (29) is the current that would flow in the nanotube in the absence of backscattering. The second term is the coherent current which oscillates with the gate voltage

$$
\begin{aligned}
\tilde{I}_{\mathrm{co}}= & c \Gamma_{1} \Gamma_{2} \cos \left[\frac{2 u g^{2} L \alpha}{\hbar^{2} v_{F}^{2}\left(1-g^{2} u^{2} / v_{F}^{2}\right)} V_{g}\right] \\
& \times \int d t \sin \left(\frac{e V_{\mathrm{sd}}}{\hbar} t\right) e^{-\tilde{C}_{\mathrm{co}}(L, t)} \sin \left[\tilde{R}_{\mathrm{co}}(L, t)\right],
\end{aligned}
$$

and the third term is the incoherent current, which is independent of the gate voltage

$$
\begin{aligned}
\tilde{I}_{\text {inco }}= & c\left\{\Gamma_{1}^{2} \sum_{\eta= \pm} \int d t \sin \left(\frac{e V_{\text {sd }}}{\hbar} t\right) e^{-\tilde{C}_{\text {inco }}^{\eta}(L, t)} \sin \left[\widetilde{R}_{\text {inco }}(L, t)\right]\right\} \\
& +c\left(\Gamma_{1} \rightarrow \Gamma_{2}, L \rightarrow-L\right)
\end{aligned}
$$

The functions $\widetilde{C}_{\mathrm{co}}, \widetilde{C}_{\text {inco }}^{ \pm}, \widetilde{R}_{\text {co }}$, and $\widetilde{R}_{\text {inco }}$ are related to the single-channel correlation functions as explained in Appen$\operatorname{dix} \mathrm{B}$.

\section{Temperature and voltage dependence in carbon nanotubes}

As in the single-channel case, we find there is a coherent part of the interference current which oscillates as a function of the gate voltage with a large period, much larger than the Fabry-Pérot oscillation period, as seen explicitly from the voltage dependence of $\widetilde{I}_{\mathrm{co}}$.

The differential conductance $\partial I_{4 \mathrm{ch}} / \partial V_{\text {sd }}$, on the other hand, displays a beating pattern, but a more complicated one than in the single-channel case, since there are four different velocities in the problem now: $v_{F} \pm u, v_{3} \approx v_{F}$, and $v_{4}$ $\approx v_{F} / g$. Figure 8 shows the differential conductance of the nanotube, $\partial I_{4 \mathrm{ch}} / \partial V_{\text {sd }}$, and its Fourier transform. From the Fourier analysis we see that clearly there are four dominant frequencies, which correspond to the four different velocities of the collective modes in the nanotube. Thus a careful observation of the large-period, and robust, Sangac interference allows, in principle, to extract the nanotube parameters, namely, the interaction strength $g$ and the velocity mismatch $u$ from the Fourier transform of the conductance as a function of bias voltage, up to temperatures much higher than the Fabry-Pérot oscillations temperatures.

The temperature dependence of the Sagnac interference in the nanotube case is qualitatively similar to the singlechannel case. In the absence of interactions $(g=1)$, the interference can be observed to the scale $T^{*}$ proportional to $v_{F} / u$; in the presence of strong interactions, however, $T^{*}$ becomes only weakly dependent on $u$. Unlike the single-channel case, $T^{*}$ in the nanotube case is also only very weakly dependent on $g$ in the range $g \leq 0.5$. This is due to the fact that only one of the four modes which diagonalize the Hamiltonian is interacting and depends on $g$. For the same reason, $T^{*}$ is higher in the case of the nanotube than in the single-channel case, i.e., the reduction in $T^{*}$ due to interactions is not as severe in the nanotube case. The temperature dependence on $u$ and $g$ is plotted in Fig. 7. In the range mentioned above, $T_{\mathrm{SAG}}^{*}$ $\approx 2.8 \frac{\hbar v_{F}}{k_{B} L} \approx 7 T_{\mathrm{FP}}^{*}$.
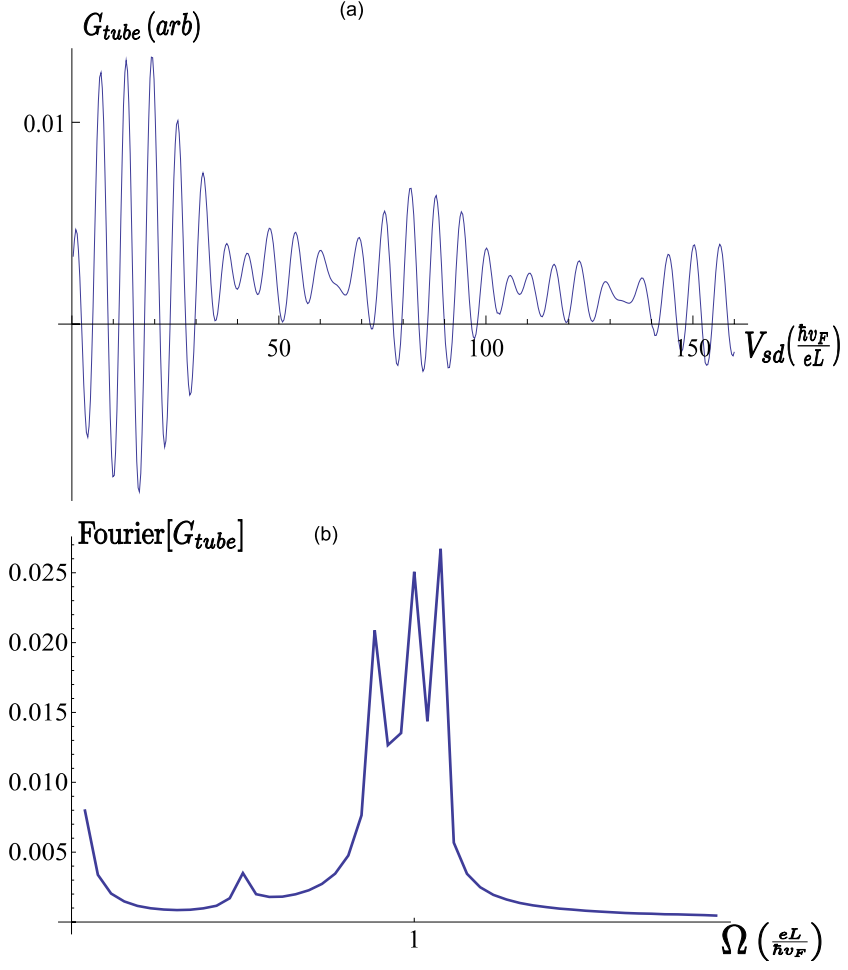

FIG. 8. (Color online) (a) Differential conductance oscillations for a nanotube, i.e., including both spins and both nodes in the spectrum, for velocity detuning $u / v_{F}=0.1$, and interaction strength $g=0.5$. In the nanotube case, the beating is due to the four voltage frequencies in the problem, $\Omega_{i}=\frac{e L}{\hbar v_{i}}$, where $v_{1 / 2}=v_{F} \pm u, v_{3} \approx v_{F}$, and $v_{4} \approx v_{F} / g$. (b) The voltage Fourier transform of the oscillations in (a) clearly displays the four dominant frequencies, $\Omega_{1-4}$, corresponding to the four velocities in the problem, and encode the nanotube parameters $v_{F}, g$, and $u / v_{F}$.

\section{SUMMARY AND CONCLUSIONS}

In this paper we investigated the conductance oscillations in carbon nanotubes due to Sagnac interference. In addition to theoretical interest in this large-period interference mode, the motivation for our study also comes from a recent experimental realization of carbon nanotube loops. ${ }^{19}$ The same effect can also be obtained without the loop geometry by internode tunneling, ${ }^{24}$ since right movers at one node of the nanotube move with the same velocity as left movers at the other node. This internode tunneling is shown in Fig. 3(c).

The source of the Sagnac conductance oscillations is the difference in the velocities of left- and right-moving excitations in a carbon nanotube when the chemical potential is tuned away from half-filling. Compared to the more familiar Fabry-Pérot oscillations, ${ }^{17}$ Sagnac oscillations are expected to have a much larger period in gate voltage, and, as we show, in noninteracting wires survive to a temperature a factor of $v_{F} / u$ higher than that required to observe Fabry-Pérot oscillations.

In interacting electronic wires, the above temperature estimation for free fermions does not apply. Our results for a single-channel Luttinger liquid are that $T_{\mathrm{SAG}}$ becomes only weakly dependent on $v_{F} / u$, although still strongly dependent on $g$. From our $g=0.5,0.25$ results, the enhancement of 
relative to the $\mathrm{FP}$ interference is roughly $T_{\mathrm{SAG}} \approx 15 g T_{\mathrm{FP}}$ in the range $u / v_{F}<0.1$.

For a strongly interacting armchair nanotube, $g \leq 0.5$, we find that $T_{\mathrm{SAG}}$ becomes not only weakly dependent on $v_{F} / u$, but also nearly independent of $g$. The Sagnac interference is expected to survive up to $T_{\mathrm{SAG}}^{*} \sim 3 \frac{\hbar v_{F}}{k_{B} L} \approx 7-8 T_{\mathrm{FP}}^{*}$. Considering that Fabry-Pérot oscillations have been observed in nanotubes up to $T=10 \mathrm{~K},{ }^{17}$ Sagnac oscillations should be observed up to about $70 \mathrm{~K}$ in nanotubes, despite the strong interactions.

There is also something to be learned from examining the behavior of the conductance as a function of the applied voltages. We saw that Sagnac oscillations would have a large period of oscillations in the applied gate voltage $V_{g}$; this period itself is a function of the gate voltage, through the dependence of the velocity difference $v_{R}-v_{L}=2 u$. Using typical values of a nanotube parameters (e.g., Ref. 19), $v_{F}$ $=8 \cdot 10^{5} \mathrm{~m} / \mathrm{s}, L=7 \mu \mathrm{m}, g=0.3$, and $\alpha=1 / 30$, the period of oscillation in the gate voltage would be $\Delta V_{g}=\frac{2 \pi \hbar v_{F}}{e L} \frac{1}{\alpha g^{2}} \frac{v_{F}}{u}$ $\approx 17 \mathrm{~V}$, consistent with the observed oscillations in Ref. 19 .

On the other hand, oscillations of the conductance as a function of the applied bias voltage $V_{\text {sd }}$ depend not only on the bare velocities, but also on the interaction strength. A Fourier transform of the Sagnac oscillations as a function of $V_{\text {sd }}$, we show, contains four different frequencies corresponding to the four different velocities in the problem, which are roughly $v_{F} \pm u, v_{F}$, and $v_{F} / g$. Using the same parameters as above we get $\Delta V_{\mathrm{sd}}=\frac{2 \pi \hbar v_{F}}{e L} \approx 0.5 \mathrm{mV}$. This period is much smaller than the bandwidth of a nanotube which is a few eV, so in principle many oscillation periods can be observed and the longer period oscillations should also be measurable, allowing the slower frequency oscillations to appear in the Fourier transform. Observation of these frequencies would allow us to read off the parameters of the nanotube, $v_{F}, u$, and $g$, at temperatures up to $T_{\mathrm{SAG}}^{*} \approx 70 \mathrm{mK}$, which is higher than the temperatures associated with Fabry-Pérot oscillations.

In the single-channel case, for noninteracting electrons, we were able to extract an analytic expression for the temperature behavior of the conductance gate voltage oscillations

$$
\frac{G_{\mathrm{co}}(T)}{G_{\mathrm{co}}(T=0)}=\left(\frac{2 \pi k_{B} L T}{\hbar v_{F}}\right)\left(\frac{u}{v_{F}}\right) \frac{1}{\sinh \left(2 \pi k_{B} T \frac{L}{\hbar v_{F}} \frac{u}{v_{F}}\right)}
$$

and it is apparent how the ratio $v_{F} / u$ directly enters the temperature scale. Unfortunately, we were so far unable to extract analytic expression for $T_{\mathrm{SAG}}$ in terms of $g$ and $u / v_{F}$ for the interacting single channel or interacting nanotube cases, in spite of the progress on the qualitative understanding our numerical results allow. Such an analytical understanding should be the focus of a future effort.

As can be observed in Figs. 1 and 2, the paths giving rise to the Sagnac interference are similar to the paths that give rise to weak-localization phenomena in $2 d$ disordered conductors. In this work we also essentially show that even in the presence of strong interactions, the interference survives. It is tempting to extrapolate from our results that weak local- ization should also survive strong interactions. This, however, is presumably true so long that scattering events are dominated by small momentum transfer. Nevertheless, our results suggest that a Luttinger liquid with charge and spin modes will still exhibit weak-localization effects, but suppressed, and only weakly dependent on the detuning between counterpropagating electrons. Therefore the magnetoresistance should also be strongly suppressed at low fields.

\section{ACKNOWLEDGMENTS}

We are indebted to Jinseong $\mathrm{Hu}$, Chetan Nayak, Yuval Oreg, Leonid Pryadko, and Jan von Delft for illuminating discussions. M.B. is grateful for support by the ONR.

\section{APPENDIX A: DIAGONALIZING THE HAMILTONIAN WITH NODE AND SPIN DEGENERACIES}

In this appendix we show how to diagonalize the Hamiltonian $H_{4 \text { ch }}$ of Eq. (24), where diagonalizing entails finding the appropriate change of basis that will transform $H_{4 \mathrm{ch}}$ to the sum of four Hamiltonians, each having a form resembling the single channel $H_{1 \mathrm{ch}}$ of Eq. (5). We also explain here the approximations we have used in our calculation.

The first step in the diagonalization of $H_{B}$ is to change the basis from the spin up or down to the spin-symmetric or antisymmetric basis at each node

$$
\theta_{a \pm}=\frac{\theta_{a \uparrow} \pm \theta_{a, \downarrow}}{\sqrt{2}}
$$

applying the same transformation to the $\phi$ 's as well. We notice that the density-density interaction term involves only the spin-symmetric fields $\theta_{a_{+}}$, hence the spin antisymmetric fields decouple and appear as two noninteracting $(g=1)$ copies of the single-channel problem, described by the Hamiltonian $H_{1 \mathrm{ch}}$, with right-moving velocity of $v_{F} \pm u$ and leftmoving velocities of $v_{F} \mp u$. These are the fields labeled with $j=1$ and $j=2$ in Eq. (25).

The Hamiltonian for the spin-symmetric fields has a similar form to our starting point Hamiltonian, $H_{4 \mathrm{ch}}$ :

$$
\begin{aligned}
H_{+}= & \frac{\hbar v_{F}}{2 \pi} \sum_{a=1,2} \int d x\left[\left(\nabla \theta_{a+}\right)^{2}+\left(\nabla \phi_{a+}\right)^{2}\right. \\
& \left.+(-1)^{a+1} 2 \frac{u}{v_{F}} \nabla \phi_{a+} \nabla \theta_{a+}\right] \\
& +\int d x 2 \lambda\left(\sum_{a=1,2} \frac{1}{\pi} \nabla \theta_{a+}\right)^{2} .
\end{aligned}
$$

In the absence of the $u$ term, $H_{+}$is easily diagonalized by taking the node symmetric and antisymmetric combinations of the fields

$$
\theta_{3 / 4}=\frac{\theta_{1+} \pm \theta_{2+}}{\sqrt{2}}
$$

The resulting diagonal Hamiltonian would be 


$$
\begin{aligned}
\left.H_{+}\right|_{u=0}= & \frac{\hbar v_{3}}{2 \pi} \int d x\left[\frac{1}{g_{3}}\left(\nabla \theta_{3}\right)^{2}+g_{3}\left(\nabla \phi_{3}\right)^{2}\right] \\
& +\frac{\hbar v_{4}}{2 \pi} \int d x\left[\frac{1}{g_{4}}\left(\nabla \theta_{4}\right)^{2}+g_{4}\left(\nabla \phi_{4}\right)^{2}\right]
\end{aligned}
$$

with $v_{3}=v_{F}, v_{4}=v_{F} / g, g_{3}=1$, and $g_{4}=g$.

When we consider $u \neq 0$, it is still possible to apply a $g$ and $u$ dependent transformation to the fields, that will restore $H_{+}$to the form in Eq. (A4), with velocities $v_{3 / 4}$ given by Eq. (26). The field mixing this transformation entails, however, considerably complicates the book keeping in our perturbative calculation. Fortunately, we can show that a good approximation is to simply set $u$ to zero in $H_{+}$when the interactions are strong, and simply use the transformation given by Eq. (A3). The first indication that this approximation is valid is that the exact velocities $v_{3 / 4}$ differ from the $u=0$ velocities by at most $1 \%$ in the entire range of parameters we are interested in, which is $u / v_{F} \leq 0.1$ and $g \leq 0.5$

Another indication that this approximation is valid comes from the analysis of the single-channel problem in Sec. II. In the single-channel case we derived exact expressions for the Sagnac interference, and found that for $g=0.5$ and $g=0.25$, the temperature dependence is only weakly dependent on $u / v_{F}$; furthermore $u$ only enters directly in the expression for the oscillation period of the conductance as a function of gate voltage, the dependence we have explicitly in our expression for the coherent current $I_{\text {co }}$ [Eq. (15)].

Finally, we can also calculate the exact combination of fields that diagonalizes $H_{+}$, and verify that indeed they are very close to the node symmetric or antisymmetric combinations for the range of $g$ and $u$ of interest. As an example, the explicit change of basis from the node symmetric or antisymmetric basis to the diagonalizing basis for $g=1 / 2$, to second order in $u / v_{F}$, is

$$
\mathrm{I}_{4 \times 4}+\left(\begin{array}{cccc}
-\frac{71}{144}\left(\frac{u}{v_{F}}\right)^{2} & 0 & 0 & \frac{2 \sqrt{2}}{3}\left(\frac{u}{v_{F}}\right) \\
0 & -\frac{89}{144}\left(\frac{u}{v_{F}}\right)^{2} & \frac{5}{3 \sqrt{2}}\left(\frac{u}{v_{F}}\right) & 0 \\
0 & \frac{-2 \sqrt{2}}{3}\left(\frac{u}{v_{F}}\right) & -\frac{29}{36}\left(\frac{u}{v_{F}}\right)^{2} & 0 \\
-\frac{5}{3 \sqrt{2}\left(\frac{u}{v_{F}}\right)} & 0 & 0 & -\frac{11}{36}\left(\frac{u}{v_{F}}\right)^{2}
\end{array}\right),
$$

We see that the matrix is close to the identity matrix $\mathbb{I}_{4 \times 4}$, since $\frac{u}{v_{F}} \ll 1$. The deviation from the identity becomes even smaller for smaller $g$. Note that for $g \approx 1$ the corresponding change of basis matrix is not close to the identity matrix and our approximation fails. This can be seen immediately for the zero interactions case, $g=1$, since when $u \neq 0$ the standard symmetric or antisymmetric combinations of fields do not diagonalize the Hamiltonian of the system.

We stress that setting $u$ to zero in $H_{+}$is simply a good numerical approximation which simplifies the calculation, and not equivalent to setting $u$ to zero in the entire problem, as $u$ still appears in spin antisymmetric part of the Hamiltonian (where $g=1$ ), and also in the gate voltage dependence.

\section{APPENDIX B: CORRELATION FUNCTIONS}

Let us now connect the explicit expressions for the coherent and incoherent currents given in Sec. II C and Sec. III, Eqs. (15) and (16), using the correlation functions defined in Sec. II B.

It is useful to define the following combination of $C^{\theta}$ :

$$
\overline{C^{\theta}(x, t)}=C^{\theta}(0,0)-C^{\theta}(x, t),
$$

and similarly for $\overline{C^{\phi}}$.
In the single-channel case discussed in Sec. II, there are only a single $\theta$ field and a single $\phi$ field, with the Hamiltonian given by Eq. (5). Since the Hamiltonian is quadratic we can easily evaluate all the equilibrium correlation functions at finite temperature, paying attention to the different time orderings that appear as a result of the two branches of the Keldysh contour. The results for finite temperature is

$$
\begin{aligned}
& \overline{C^{\theta}(x, t)}= \frac{g}{4}\left\{\log \left[\frac{\beta v_{L}}{\pi \delta} \sinh \left(\frac{\pi\left(x+v_{L} t-i \delta\right)}{\beta v_{L}}\right)\right]\right. \\
&+\log \left[\frac{\beta v_{R}}{\pi \delta} \sinh \left(\frac{\pi\left(x-v_{R} t+i \delta\right)}{\beta v_{R}}\right)\right] \\
&+(x \rightarrow-x, t \rightarrow-t)\} ; \\
& R^{\theta}(x, t)=-\frac{\pi}{2} g\left[\Theta(x) \Theta\left(t-\frac{x}{v_{R}}\right)+\Theta(-x) \Theta\left(t-\frac{|x|}{v_{L}}\right)\right],
\end{aligned}
$$

where $\delta$ is a short-distance cutoff, $v_{R / L}=v_{F} / g \pm u$, and $\Theta(x)$ is the step function. As mentioned in Ref. 28, it is important to remember that the step functions are not infinitely sharp, 
and have a transition width of order $a$, the cutoff. The functions $\overline{C^{\phi}(x, t)}$ and $R^{\phi}(x, t)$ are obtained from $\overline{C^{\theta}(x, t)}$ and $R^{\theta}(x, t)$ by replacing the prefactor $g$ with $\frac{1}{g}$. The function $C^{\theta \phi}$ is given in Eq. (12), and

$$
R^{\theta \phi}(x, t)=-\frac{\pi}{2} \Theta(t)\left[\Theta(x) \Theta\left(x-v_{R} t\right)-\Theta(-x) \Theta\left(|x|-v_{L} t\right)\right] .
$$

The currents are expressed in integrals over complicated combinations of such correlation functions. For example, the coherent part of the current, given by Eq. (15), involves the following combinations

$$
\begin{aligned}
C_{\mathrm{co}}(L, t)= & 2 C^{\theta \phi}(L, t)-2 C^{\theta \phi}(-L, t)+2 \overline{C^{\theta}(0, t)}-2 \overline{C^{\theta}(L, 0)} \\
& +\overline{C^{\theta}(L, t)}+\overline{C^{\theta}(-L, t)}+2 \overline{C^{\phi}(L, 0)}-2 \overline{C^{\phi}(0, t)} \\
& +\overline{C^{\phi}(L, t)}+\overline{C^{\phi}(-L, t)}
\end{aligned}
$$

and

$$
\begin{aligned}
R_{\mathrm{co}}(L, t)= & R^{\theta \phi}(L, t)-R^{\theta \phi}(-L, t)+R^{\theta}(0, t)+\frac{1}{2} R^{\theta}(L, t)+\frac{1}{2} R^{\theta}( \\
& -L, t)-R^{\phi}(0, t)+\frac{1}{2} R^{\phi}(L, t)+\frac{1}{2} R^{\phi}(-L, t) .
\end{aligned}
$$

The corresponding functions for the incoherent current are

$$
\begin{aligned}
C_{\mathrm{inco}}^{ \pm}(L, t)= & \pm\left[2 C^{\theta \phi}(L, t)-2 C^{\theta \phi}(-L, t)\right]+2 \overline{C^{\theta}(0, t)} \\
& -2 \overline{C^{\theta}(L, 0)}+\overline{C^{\theta}(L, t)}+\overline{C^{\theta}(-L, t)}+2 \overline{C^{\phi}(L, 0)} \\
& +2 \overline{C^{\phi}(0, t)}-\overline{C^{\phi}(L, t)}-\overline{C^{\phi}(-L, t)}
\end{aligned}
$$

and

$$
\begin{aligned}
R_{\text {inco }}(L, t)= & R^{\theta}(0, t)+\frac{1}{2} R^{\theta}(L, t)+\frac{1}{2} R^{\theta}(-L, t)+R^{\phi}(0, t) \\
& -\frac{1}{2} R^{\phi}(L, t)-\frac{1}{2} R^{\phi}(-L, t) .
\end{aligned}
$$

In a carbon nanotube there are four channels, rather than a single one. In the noninteracting case, $g=1$, all these channels are independent and we would recover the results of the single channel. Equations (B5) and (B6) still apply for this case. When $g \neq 1$, the different channels are coupled through the interaction, and we must find the correct combinations of the fields $\theta_{i \sigma}$ and $\phi_{i \sigma}$ which decouple and therefore diagonalize the Hamiltonian. These combinations are discussed in Appendix A. This change of basis is in general a function of $u / v_{F}$ and $g$, and it mixes the $\theta$ and $\phi$ fields, which in turn complicates the functions $C_{\mathrm{co}}$ and $R_{\mathrm{co}}$ further. Luckily, the interactions in carbon nanotubes are strong, $g \approx 0.3$, and in that range, the change in basis is very close to the usual spin or node symmetric or antisymmetric change in basis. If we approximate the diagonalizing fields by these symmetric or antisymmetric combinations, then Eqs. (B5) and (B6) would apply provided we make the following substitutions:

$$
\overline{C^{\theta}(x, t)} \rightarrow \frac{1}{4} \sum_{j=1 . .4} \overline{C^{\theta_{j}}(x, t)},
$$

where each $\theta_{j}$ has a different set of values for $v_{R}, v_{L}$, and $g$ to be used in Eq. (B2). The fields $\theta_{1}$ and $\theta_{2}$ correspond to the spin asymmetric combinations, which decouple from the interaction, and hence have $g=1$, and velocities $v_{R}=v_{F} \pm u$ and $v_{L}=v_{F} \mp u$. The fields $\theta_{3}$ and $\theta_{4}$ both have the same left- and right-mover velocities, $v_{3}$ and $v_{4}$, respectively, given by Eq. (26), and interaction parameters 1 and $g$, respectively.
${ }^{1}$ G. Sagnac, Acad. Sci., Paris, C. R. 157, 708 (1913).

${ }^{2}$ Y. Japha, O. Arzouan, Y. Avishai, and R. Folman, Phys. Rev. Lett. 99, 060402 (2007).

${ }^{3}$ J. Xia, E. Schemm, G. Deutscher, S. A. Kivelson, D. A. Bonn, W. N. Hardy, R. Liang, W. Siemons, G. Koster, M. M. Fejer, and A. Kapitulnik, Phys. Rev. Lett. 100, 127002 (2008).

${ }^{4}$ H. Förster, S. Pilgram, and M. Büttiker, Phys. Rev. B 72, 075301 (2005).

${ }^{5}$ F. E. Camino, W. Zhou, and V. J. Goldman, Phys. Rev. B 72, 155313 (2005).

${ }^{6}$ W. G. van der Wiel, Y. V. Nazarov, S. De Franceschi, T. Fujisawa, J. M. Elzerman, E. W. G. M. Huizeling, S. Tarucha, and L. P. Kouwenhoven, Phys. Rev. B 67, 033307 (2003).

${ }^{7}$ B. J. van Wees, L. P. Kouwenhoven, C. J. P. M. Harmans, J. G. Williamson, C. E. Timmering, M. E. I. Broekaart, C. T. Foxon, and J. J. Harris, Phys. Rev. Lett. 62, 2523 (1989).

${ }^{8}$ E. Buks, R. Schuster, M. Heiblum, D. Mahalu, and V. Umansky, Nature (London) 391, 871 (1998).

${ }^{9}$ D. E. Feldman and A. Kitaev, Phys. Rev. Lett. 97, 186803 (2006).

${ }^{10}$ A. Stern and B. I. Halperin, Phys. Rev. Lett. 96, 016802 (2006).
${ }^{11}$ P. Bonderson, A. Kitaev, and K. Shtengel, Phys. Rev. Lett. 96, 016803 (2006).

${ }^{12}$ M. Sigrist, Thomas Ihn., K. Ensslin, M. Reinwald, and W. Wegscheider, Phys. Rev. Lett. 98, 036805 (2007).

${ }^{13}$ A. Yacoby, M. Heiblum, D. Mahalu, and H. Shtrikman, Phys. Rev. Lett. 74, 4047 (1995).

${ }^{14}$ M. Bockrath, D. H. Cobden, J. Lu, A. G. Rinzler, R. E. Smalley, L. Balents, and P. L. McEuen, Nature (London) 397, 598 (1999).

${ }^{15}$ C. Kane, L. Balents, and M. P. A. Fisher, Phys. Rev. Lett. 79, 5086 (1997).

${ }^{16}$ Z. Yao, H. W. C. Postma, L. Balents, and C. Dekker, Nature (London) 402, 273 (1999).

${ }^{17}$ W. Liang, M. Bockrath, D. Bozovic, J. H. Hafner, M. Tinkham, and H. Park, Nature (London) 411, 665 (2001).

${ }^{18}$ C. S. Peça, L. Balents, and K. J. Wiese, Phys. Rev. B 68, 205423 (2003).

${ }^{19}$ G. Refael, J. Heo, and M. Bockrath, Phys. Rev. Lett. 98, 246803 (2007).

${ }^{20}$ V. Fernandez, A. Iucci, and C. Nan, Eur. Phys. J. B 30, 53 (2002). 
${ }^{21}$ E. Abrahams, P. W. Anderson, D. C. Licciardello, and T. V. Ramakrishnan, Phys. Rev. Lett. 42, 673 (1979).

${ }^{22}$ L. P. Gor'kov, D. Khmel'nitzkii, and A. I. Larkin, Pis'ma Zh. Eksp. Teor. Fiz. 30, 248 (1979).

${ }^{23}$ B. L. Altshuler, A. G. Aronov, and P. A. Lee, Phys. Rev. Lett. 44, 1288 (1980).

${ }^{24}$ J. Jiang, J. Dong, and D. Y. Xing, Phys. Rev. Lett. 91, 056802 (2003).
${ }^{25}$ L. V. Keldysh, Zh. Eksp. Teor. Fiz. 47, 1515 (1964) [Sov. Phys. JETP 20, 1018 (1965)].

${ }^{26}$ J. Rammer and H. Smith, Rev. Mod. Phys. 58, 323 (1986).

${ }^{27}$ C. L. Kane and M. P. A. Fisher, Phys. Rev. B 46, 15233 (1992).

${ }^{28}$ P. Recher, N. Y. Kim, and Y. Yamamoto, Phys. Rev. B 74, 235438 (2006).

${ }^{29}$ Y. Oreg and A. M. Finkel'stein, Phys. Rev. Lett. 74, 3668 (1995). 